\title{
Selbstständigkeit dient der Professionalisierung Sozialer Arbeit
} Auf dem Weg zu einem »Erbringungsmix « sozialer Dienstleistungen

\author{
Monika Köppel
}

Social services provided within independent settings are increasingly meaningful as a new form of service provision. The main reason is financial regression going hand in hand with an increasing need for social provisions.

La prestation de services sociaux proposée dans un cadre du travail indépendant est de plus en plus significative en terme d'innovation. La raison principale réside dans la récession financière qui va de pair avec la demande croissante de services sociaux.

Monika Köppel ist Diplom-Sozialpädagogin und Kooperationsmanagerin M.A. Derzeit promoviert sie an der Ludwig-Maximilans-Universität München. Sie ist selbstständig tätig in einer eigenen Praxis für Sozialtherapie und psychosoziale Beratung.
In Selbstständigkeit erbrachte soziale Dienstleistungen gewinnen als eine für die Soziale Arbeit innovative Form der Leistungserbringung an Bedeutung. Hauptgründe sind finanzielle Regressionen bei gleichzeitig steigendem Bedarf sozialer Unterstützungsleistungen.

Soziale Arbeit avanciert aufgrund der Zunahme gesamtgesellschaftlicher Risiken zum »Risikogewinner" (1), was durchaus auch eine ökonomische Betrachtungsweise darstellt. Das macht es erforderlich, dass sich die gesamte Profession konstruktiv mit dieser Thematik auseinandersetzt und ihre Strukturen dahingehend öffnet. Insbesondere da sich hieraus neue Chancen ergeben, ökonomische Zielsetzungen mit ethischen Zielsetzungen $\mathrm{zu}$ verbinden, neue Handlungsspielräume für die Profession und deren Klientel zu sichern und den bislang wenig erfolgreichen Prozess der Professionalisierung positiv voranzutreiben.

Generell lässt sich der hohe Stellenwert selbstständiger Leistungserbringung und der Grundgedanke, dass insbesondere Selbstständigkeit als Leitbild von Professionalisierungsprozessen $\mathrm{zu}$ betrachten ist, bis zu Parsons zurückverfolgen. Er verknüpft erstmalig motivationale Aspekte akademischer Akteure, wie etwa das Verfolgen »höherer Ziele«, mit der Notwendigkeit und dem Streben nach (Handlungs-) Autonomie, wie sie vor allem in Selbstständigkeit zu erreichen ist und bezeichnet diese als kennzeichnendes Merkmal und als Leitbild von Professionalisierungsprozessen. Parsons vertritt die Ansicht, vor allem (Handlungs-) Autonomie diene dazu, als Zeichen unhinterfragter Kompetenz, Machtverhältnisse auszubauen und durch dessen positive Beeinflussung den gesellschaftlichen Anerkennungsprozess zu sichern. (2)

Soziale Arbeit tut sich mit solchen Einsichten und (Selbst-) Entwicklungstendenzen traditionell schwer, wie etwa mit dem Einbezug vermehrter selbstständiger Leistungserbringung oder dem verstärkten Einbezug nicht marginalisierter Klienten in ihre Leistungsfelder und somit einer Verknüpfung monetärer Interessen mit ethischen Zielsetzungen. Soziale Arbeit wird sowohl in den Köpfen der professionellen Akteure, als auch innerhalb der Gesellschaft immer noch verknüpft, mit unausgesprochenen oder unbewussten Implikationen, die das Selbst- und Fremdbild der Profession beeinflussen und hinsichtlich ihrer Weiterentwicklung und Adaption an sich ändernde gesellschaftliche Lagen und Bedarfe negativ beeinflussen. Immer noch schwingen Vorstellungen des barmherzigen Samariters in den Köpfen der aus der freien Liebestätigkeit und Fürsorge entsprungenen Profession mit.

Diese Vorstellung hat zur Folge, dass eine Verknüpfung ökonomischer Interessen und ethischer und Zielsetzungen in Frage gestellt wird. Dabei bleibt unberücksichtigt, dass Soziale Arbeit in erster Linie ein Beruf ist, der den Ausübenden helfen soll, ihren Lebensunterhalt zu sichern und der erst in zweiter Linie die erfreuliche und innerhalb unserer Gesellschaft sicherlich immer notwendiger werdende Möglichkeit mit sich bringt, Menschen zu einem gelingenderen Leben zu verhelfen.

Mit Thiersch und Grunwald lässt sich daher in diesem Zusammenhang Folgendes festhalten: Es macht wenig Sinn, an alten Strukturen festzuhalten, wenn diese ihre Wirksamkeit verloren haben und den aktuellen Anforderungen nicht mehr genügen. (3) Vielmehr besteht die Notwendigkeit der Adaption und der Öffnung dieser Strukturen zu flexibleren, nutzerorientierten und bedarfsgerechteren Formen der Leistungserbringung. Sinnvoll erscheint in diesem Zusammenhang eine professionell koordinierte und initiierte Mischung aus staatlichen Leistungen, marktwirtschaftlichen Leistungen und naturwüchsigen, möglicherweise reaktivierten Solidarnetzen, bei vorange- 
gangener und wenn notwendig, begleitender Befähigung der Subjekte.

In diesem Zusammenhang stehen selbstständig tätige Akteure innerhalb Sozialer Arbeit in der Pflicht, ihre Kompetenzen und Machtverhältnisse zu bündeln, die eigenen Fähigkeiten sowohl politischen Entscheidungsträgern als auch der breiten Öffentlichkeit transparent und zugänglich zu machen und damit den Professionalisierungsprozess Sozialer Arbeit voranzutreiben. (4)

Dass dies den individuellen Kompetenz- und Machtrahmen jedes Einzelnen wie der gesamten Profession erweitert, ist dabei nicht nur als eine begrüßenswerte Randerscheinung zu betrachten, sondern als dringliche Notwendigkeit, um sich als selbstbewusster Leistungserbringer auf dem Markt der Sozial- und Gesundheitsdienstleistungen zu präsentieren und sich im Daseinskampf konkurrierender Professionen dauerhaft zu behaupten. Dabei ist es notwendig, den Umgang mit dieser Macht professionell zu erlernen, mit dem Ziel, selbstbestimmte und wissensbasierte Aufträge für die gesamte Profession zu generieren, da diese für deren künftige Entwicklung von größter Bedeutung sind, um sich nicht aufgrund fremd gesteuerter Zuweisungen selbst zu beschränken. (5)

Dass dies nicht ausschließlich selbstständig tätige Akteure betrifft, sondern die gesamte Profession, macht die zunehmende Regressionsfreudigkeit sozialer Leistungen innerhalb der bundesdeutschen Sozialstaatlichkeit deutlich. In deren Folge kommt es zu immer weitreichenderen Kürzungen und kompletten Streichungen öffentlich finanzierter sozialer Leistungen, wodurch der Stellenwert selbstständig erbrachter Dienstleistungen mehr denn je an Bedeutung gewinnt. Deutlich wird dies bei einer vergleichenden Betrachtung des gesellschaftlichen Stellenwertes hoch etablierter Professionen, welche diese Form der Leistungserbringung und damit die Berücksichtigung monetärer Ziele schon lange und erfolgreich praktizieren und damit ihre professionseigenen Machtpotenziale sukzessive ausgebaut und gesichert haben. Zu denken ist in diesem Zusammenhang beispielsweise an Mediziner, Psychologen und Juristen.

Diesen Professionen ist gemeinsam, dass sie ihre Dienstleistungen, unter dem legitimen Einbezug ökonomischer Interessen und Zielsetzungen, in einem »Er- bringungsmix « vorhalten, der sich einerseits auf die zu erreichenden Zielkunden bezieht und andererseits auf die Erbringungsstruktur selbst. Während sich die Kunden differenzieren lassen, in marginalisiertes und zahlungskräftiges Klientel, lassen sich die Erbringungsstrukturen in ähnlicher Weise in »Angestelltenstrukturen « und "Selbstständigenstrukturen « differenzieren. Grund- und Pflichtleistungen werden dabei vornehmlich von Angestellten erbracht, während Selbstständige für zusätzliche Wahlleistungen zuständig sind.

Jedoch zeichnet sich aufgrund der ökonomischen Regressionen in allen gesellschaftlichen Bereichen, auch hier mehr und mehr eine allmähliche Vermischung dieser Strukturen ab. Festzuhalten bleibt der Vorteil dieser Strukturen, der vor allem darin besteht, dass sich die Professionen eine ökonomische Basis schaffen, die Aspekten (sozial-) anwaltschaftlicher Bestrebungen oder Verpflichtungen keineswegs entgegenstehen, da diese unter dem Gesichtspunkt der Bedarfsgerechtigkeit gleichermaßen Berücksichtigung finden. Schramm spricht in diesem Zusammenhang von einer Verknüpfung ethischer und ökonomischer Zielsetzungen, in deren Folge sich Freiräume schaffen lassen, welche die Verfolgung ethischer Ziele vielfach sogar erst ermöglichen. (6)

Als positive Begleiterscheinung dieser Vorgehensweise lässt sich außerdem eine zunehmende Entstigmatisierung der in Anspruch genommenen Leistungen und damit eine Normalisierung der Inanspruchnahme feststellen. Dies macht deutlich: Eine Verknüpfung monetärer Aspekte mit ethischen Aspekten bringt nicht nur den Vorteil der eigenen Existenzsicherung der jeweiligen Profession und der Ausweitung ihrer gesellschaftlichen Anerkennung mit sich, sondern außerdem den Nutzern dieser Leistungen den Vorteil einer Normalisierung ihrer Inanspruchnahme.

Die gleichzeitige Notwendigkeit und der Nutzen selbstständiger Leistungserbringung wird auch von den Hochschulen mehr und mehr registriert und findet dahingehend Berücksichtigung, dass Versuche unternommen werden Studenten verstärkt auf diese Form der Leistungserbringung vorzubereiten. Als problematisch erweist sich dabei momentan jedoch die Tatsache, dass es nur wenige Institutionen gibt, die fundierte Kenntnisse über die spezifischen Anforderungen solcher Existenzgründungen in Sozial- und Gesundheitsdiensten besitzen. Zudem ist die empirische Datenlage in diesem Bereich noch zu dünn, um Kennzahlen zur internen und externen Beurteilung und Steuerung zu entwickeln.

\section{Anmerkungen}

(1) Vgl. Rauschenbach 1992

(2) Vgl. Parsons 1973

(3) Vgl. Thiersch/Grunwald 2001

(4) Vgl. Köppel 2003

(5) Vgl. Staub-Bernasconi 1995

(6) Vgl. Schramm 1998

\section{Literatur}

Köppel, Monika (2003): Salutogenese und Soziale Arbeit. Lage. Verlag Hans Jakobs.

Parsons, Talcott (1973): Soziologische Theorie. 3. Auflage. Soziologische Texte 15. Darmstadt, Neuwied. Luchterhand Verlag.

Schramm, Michael (1998): Geld und Moral. Zur Strategie der Sozialethik. In: Religionsunterricht an höheren Schulen 41. Westermann Verlag. S. 224-229.

Rauschenbach, Thomas (1992): Soziale Arbeit und Soziales Risiko. In: Rauschenbach, T.; Gängler, H. (Hg.): Soziale Arbeit und Erziehung in der Risikogesellschaft. Neuwied. Luchterhand Verlag. S. 25-60.

Staub-Bernasconi, Silvia (1995): Das fachliche Selbstverständnis Sozialer Arbeit. Wege aus der Bescheidenheit. Soziale Arbeit als Human Rights Profession. In: Wendt, W. (Hg.): Soziale Arbeit im Wandel ihres Selbstverständnisses. Freiburg im Breisgau. Lambertus Verlag. S. 57-104.

Thiersch, Hans; Grunwald, Klaus (2001): Lebensweltorientierung. In: Otto, Hans-Uwe; Thiersch, Hans (Hg.): Handbuch Sozialarbeit Sozialpädagogik. 2. Auflage. Neuwied, Kriftel. Luchterhand Verlag. S. 1136-1148. 